Article

\title{
Stark Broadening of Cr III Spectral Lines: DO White Dwarfs
}

\author{
Milan S. Dimitrijević 1,2,+ (id) and Abhishek Chougule ${ }^{1,3}$ \\ 1 Astronomical Observatory, Volgina 7, 11060 Belgrade 38, Serbia; Abhishek.Chougule@student.uibk.ac.at \\ 2 LERMA, Observatoire de Paris, PSL Research University, CNRS, Sorbonne Universities, UPMC, Paris 06, \\ 5 Place Jules Janssen, 92195 Meudon CEDEX, France \\ 3 Institut für Astro- und Teilchenphysik, Universität Innsbruck, Technikerstr. 25/8, A-6020 Innsbruck, Austria \\ * Correspondence: mdimitrijevic@aob.rs or milan.dimitrijevic@obspm.fr; Tel.: +381-64-297-8021 \\ + Current address: Astronomical Observatory, Volgina 7, 11060 Belgrade 38, Serbia.
}

Received: 7 March 2018; Accepted: 26 March 2018; Published: 3 April 2018

check for updates

\begin{abstract}
Using the modified semiempirical method of Dimitrijević and Konjević, Stark widths have been calculated for six Cr III transitions, for an electron density of $10^{17} \mathrm{~cm}^{-3}$ and for temperatures from 5000-80,000 K. Results have been used for the investigation of the influence of Stark broadening on spectral lines in cool DO white dwarf atmospheres. Calculated Stark widths will be implemented in the STARK-B database, which is also a part of the Virtual Atomic and Molecular Data Center (VAMDC).
\end{abstract}

Keywords: Stark broadening; line profiles; atomic data; DO white dwarfs; stellar spectra

\section{Introduction}

Stark broadening data are very useful for a number of applications, such as laboratory plasma diagnostics, the investigation and modelling of various plasmas in technology and inertial fusion, as well as for the research of laser-produced plasmas, and particularly in astrophysics, primarily for stellar plasma modelling, abundance determination, and stellar spectra analysis and synthesis.

Lines of chromium in various ionization stages are present and often observed in stellar spectra. Underhill [1] identified a great number of Cr II and Cr III lines, as well as a probable Cr IV line in the spectrum of B5 Ia type star $\eta$ CMa recorded by Orbiting Astronomical Observatory OAO-3, also known as Copernicus. Underhill [2] also found a large number of Cr II and Cr III lines and concluded that Cr IV lines are probably present in the spectra of $\tau$ Her (B5 IV) and $\zeta$ Dra (B6 III), also obtained by the spectrometer on OAO-3. A feature of Cr III was found by Fahey et al. [3] in the spectrum of $\beta$ Cephei obrtained by OAO-2. A number of Cr II and Cr III lines were also identified by Bauer and Bennett [4] in the spectrum of $\zeta$ Aurigae binary system 31 Cygni (K4 Ib + B4 V), obtained by the FUSE (Far Ultraviolet Spectroscopic Explorer) satellite. Generally, the iron group elements contribute very significantly to the observed opacity in stellar atmospheres [5], and doubly ionized spectra of this group -including Cr III-dominate the UV spectra of hot B stars [6-8].

$\mathrm{Cr}$ II lines have also been observed in the spectra of chemically peculiar A-type stars, where Stark broadening is the dominant pressure broadening mechanism, like 7 Sex [9] and $\phi$ Aqu [10], where $28 \mathrm{Cr}$ II spectral lines have been identified. Chromium lines have been observed and in A-type star spectra of $o$ Peg [11] and Przybylski's star [12]. In Ae/Be Herbig Star V 380 Ori, where Shevchenko [13] found $25 \mathrm{Cr}$ II lines, ionized chromium spectral lines are before Fe II and Ti II in number and intensity. Ionized chromium lines were also found, e.g. in $\alpha$ UMi (Polaris) and HR 7308 by Andrievsky et al. [14], and in XX Oph [15], yet in 1951, Merrill [16] found 58 emission Cr II lines. With the development of high-resolution spectrographs, the assumption of the homogeneous distribution of an element in stellar atmosphere becomes less adequate, for example Babel and Lanz [17] investigated the 
influence of stratification on chromium lines in the spectrum of chemically peculiar Ap 53 Cam star, and Dimitrijević, M. S. et al. [18] in the Ap HD 133972.

Chromium in various ionization stages from $\mathrm{Cr}$ I to $\mathrm{Cr}$ VI has been observed and in the spectra of white dwarfs, where the Stark broadening is often the dominant broadening mechanism in wide layers of the atmosphere [19-27]. For example, Cr I has been found by Gianninas et al. [28] in an extremely low mass white dwarf in the SDSS J074511.56+194926.5 binary system and by Dufour et al. [29] in the spectrum of DZ white dwarf G165-7; Cr II spectral lines were identified by Klein et al. [30] in the spectrum of white dwarf GD 40, observed on the Keck I telescope at Mauna Kea Observatory; and, for example, Cr V and Cr VI by Rauch et al. [31] in the spectrum of the white dwarf central star of Sh 2-216.

In spite of the importance of chromium, theoretical data on Stark broadening parameters are scarce. Dimitrijević, M. S. et al. [32] used the semiclassical perturbation method-SCP [33-35] - to calculate electron-, proton-, and ionized helium-impact widths and shifts for nine $\mathrm{Cr}$ I spectral lines from the $4 \mathrm{p}^{7} \mathrm{P}^{0}-4 \mathrm{~d}^{7} \mathrm{D}$ multiplet and used the obtained results to investigate the influence of Stark broadening on line shapes in the spectrum of Cr-rich Ap star $\beta$ CrB atmosphere. By using the SCP method, Dimitrijević, M. S. et al. [18] obtained electron-, proton-, and ionized helium-impact widths and shifts for spectral lines of seven $\mathrm{Cr}$ II multiplets belonging to $4 \mathrm{~s}-4 \mathrm{p}$ transitions. They applied obtained Stark broadening parameters to the analysis of $\mathrm{Cr}$ II line profiles observed in the spectrum of $\mathrm{Cr}$-rich Ap-type star HD 133792, and found that the Stark broadening mechanism is very important and should be taken into account. Simić et al. [25] calculated Stark broadening parameters for an additional nine resonance $\mathrm{Cr}$ II multiplets from $3 \mathrm{~d}^{5}-3 \mathrm{~d}^{4} 4 \mathrm{p}$ transition array using the same method. For $\mathrm{Cr}$ I and $\mathrm{Cr}$ II there are also the estimates of Lakićević [36], based on regularities and systematic trends, for Stark width and shift of resonance lines Cr I $4289.7 \AA$ and Cr II 2065.5 A. A large number of Stark-damping constants from chromium lines may also be found in Kurucz [37]. For the Stark broadening of Cr III, $\mathrm{Cr} \mathrm{IV}$, and $\mathrm{Cr} \mathrm{V}$ lines, there is no data in the literature. Electron-, proton-, and ionized helium-impact widths and shifts only exist for two multiplets of Cr VI [38].

Experimental data exist only for Cr II lines. Rathore et al. [39] experimentally determined the widths and shifts of three lines within the Cr II multiplet $4 \mathrm{~s}^{4} \mathrm{D}-4 \mathrm{p}^{4} \mathrm{~F}^{o}$ and Aguilera et al. [40] measured the widths and shifts of $83 \mathrm{Cr}$ II lines within 45 multiplets.

As we can see, data for the Stark broadening of Cr III lines observed and present in stellar atmospheres are completely missing. Data on the energy levels of $\mathrm{Cr}$ III are not complete, and it is not possible to perform a more sophisticated semiclassical perturbation calculation. So, in this work we will calculate full widths at half intensity maximum (FWHM) due to collisions with surrounding electrons for Cr III spectral lines, using the modified semiempirical method (MSE) [41-43]. As an example of the importance of of Stark broadening data for stellar spectra analysis and synthesis and stellar plasma investigation and modelling, we will compare Stark and thermal Doppler broadening importance using two models of white dwarf atmospheres.

\section{The Modified Semiempirical Method}

For the calculations of Stark full width at half-intensity maximum (FWHM), the modified semiempirical (MSE) approach [41] is used. It is explained in detail in [41-43], and only basic expressions needed for the understanding of the calculation method will be given here. The FWHM of an isolated ion line is given by the following equation:

$$
\begin{gathered}
w_{M S E}=N \frac{4 \pi}{3 c} \frac{\hbar^{2}}{m^{2}}\left(\frac{2 m}{\pi k T}\right)^{1 / 2} \frac{\lambda^{2}}{\sqrt{3}} \cdot\left\{\sum_{\ell_{i} \pm 1} \sum_{L_{i^{\prime}} I_{i^{\prime}}} \vec{\Re}_{\ell_{i}, \ell_{i} \pm 1}^{2} \widetilde{g}\left(x_{\ell_{i}, \ell_{i} \pm 1}\right)+\right. \\
\left.\sum_{\ell_{f} \pm 1} \sum_{L_{f^{\prime}} I_{f^{\prime}}} \vec{\Re}_{\ell_{f}, \ell_{f} \pm 1}^{2} \widetilde{g}\left(x_{\ell_{f}, \ell_{f} \pm 1}\right)+\left(\sum_{i^{\prime}} \vec{\Re}_{i^{\prime}}^{2}\right)_{\Delta n \neq 0} g\left(x_{n_{i}, n_{i}+1}\right)+\left(\sum_{f^{\prime}} \vec{\Re}_{f f^{\prime}}^{2}\right)_{\Delta n \neq 0} g\left(x_{n_{f}, n_{f}+1}\right)\right\} .
\end{gathered}
$$


Here, $i$ and $f$ are for initial and final levels, respectively, $\vec{\Re}_{\ell_{k}, \ell_{k^{\prime}}}^{2}, k=i, f$ is the square of the matrix element, and

$$
\left(\sum_{k^{\prime}} \vec{\Re}_{k k^{\prime}}^{2}\right)_{\Delta n \neq 0}=\left(\frac{3 n_{k}^{*}}{2 Z}\right)^{2} \frac{1}{9}\left(n_{k}^{* 2}+3 \ell_{k}^{2}+3 \ell_{k}+11\right)
$$

(in Coulomb approximation).

In Equation (1),

$$
x_{l_{k}, l_{k^{\prime}}}=\frac{E}{\Delta E_{l_{k}, l_{k^{\prime}}}}, k=i, f,
$$

where $E=\frac{3}{2} k T$ is the electron kinetic energy and $\Delta E_{l_{k}, l_{k^{\prime}}}=\left|E_{l_{k}}-E_{l_{k^{\prime}}}\right|$ is the energy difference between levels $l_{k}$ and $l_{k} \pm 1(k=i, f)$,

$$
x_{n_{k}, n_{k}+1} \approx \frac{E}{\Delta E_{n_{k}, n_{k}+1}},
$$

where for $\Delta n \neq 0$ the energy difference between energy levels with $n_{k}$ and $n_{k}+1, \Delta E_{n_{k}, n_{k}+1}$, is estimated as $\Delta E_{n_{k}, n_{k}+1} \approx 2 Z^{2} E_{H} / n_{k}^{* 3}$. With $n_{k}^{*}=\left[E_{H} Z^{2} /\left(E_{i o n}-E_{k}\right)\right]^{1 / 2}$ is denoted the effective principal quantum number, $Z$ is the residual ionic charge (e.g., $Z=1$ for neutral atoms), $E_{i o n}$ is the appropriate spectral series limit, $N$ and $T$ are electron density and temperature, respectively, while $g(x)[44]$ and $\widetilde{g}(x)[41]$ are the corresponding Gaunt factors.

\section{Results and Discussion}

The needed set of atomic energy levels was taken from Sugar and Corliss [45] (see also NIST database [46]), and the dipole matrix elements for considered transitions were calculated within Coulomb approximation [47]. Stark widths of Cr III spectral lines were calculated by using the modified semiempirical method [41] (see also the review of innovations and applications in [43]). The obtained results are shown in Table 1 for a perturber density of $10^{17} \mathrm{~cm}^{-3}$ and temperatures from $5000 \mathrm{~K}$ up to $80,000 \mathrm{~K}$.

The presented data for Stark broadening of Cr III lines are the first, so there is no other experimental or theoretical data for comparison.

The Stark broadened shape $F(\omega)$ of an isolated spectral line has Lorentzian form, and can be represented as:

$$
F(\omega)=\frac{W / 2 \pi}{\left(\omega-\omega_{i f}-d\right)^{2}+(W / 2)^{2}}
$$

Here,

$$
\omega_{i f}=\frac{E_{i}-E_{f}}{\hbar} .
$$

In the upper equation $E_{i}$ and $E_{f}$ are energies of the initial and final states, while Stark FWHM (W) and shift $(d)$ are in angular frequency units. For the difference of Stark width, were contributions of all term in Equation (1) are positive, for shift the analogous contributions of particular terms may be positive and negative. Additionally, the theoretical accuracy is smaller due to mutual cancellations of various terms. For $s-p$ transitions considered here, the contribution of the lower $s$ state is negative and the shift will be smaller than width, so it could be neglected. In order to obtain the line shape, one should transform results from Table 1 from $\AA$-units to angular frequency units using the following expression:

$$
W(\AA)=\frac{\lambda^{2}}{2 \pi c} W\left(s^{-1}\right),
$$

where $c$ is the speed of light. 
Table 1. Full Width at Half intensity Maximum (FWHM, A) for Cr III spectral lines, for a perturber density of $10^{17} \mathrm{~cm}^{-3}$ and temperatures from $5000 \mathrm{~K}$ to $80,000 \mathrm{~K}$. Calculated wavelength $(\lambda)$ of the transitions (in $\AA$ ) is also given. $3 \mathrm{kT} / 2 \Delta \mathrm{E}$ is the ratio of the energy of free electron and of the energy difference to the closest perturbing level for $\mathrm{T}=10,000 \mathrm{~K}$.

\begin{tabular}{|c|c|c|}
\hline Transition & $\mathrm{T}(\mathrm{K})$ & FWHM (Å) \\
\hline \multirow[t]{2}{*}{ CrIII $3 d^{3}\left({ }^{4} F\right) 4 s^{5} F-3 d^{3}\left({ }^{4} F\right) 4 p^{5} F^{o}$} & 5000 & $0.723 \mathrm{E}-01$ \\
\hline & 10,000 & $0.511 \mathrm{E}-01$ \\
\hline$\lambda=2121.8 \AA$ & 20,000 & $0.361 \mathrm{E}-01$ \\
\hline \multirow{2}{*}{$3 \mathrm{kT} / 2 \Delta \mathrm{E}=0.234$} & 40,000 & $0.256 \mathrm{E}-01$ \\
\hline & 80,000 & $0.181 \mathrm{E}-01$ \\
\hline \multirow[t]{2}{*}{ CrIII $3 d^{3}\left({ }^{4} F\right) 4 s^{5} F-3 d^{3}\left({ }^{4} F\right) 4 p^{5} G^{o}$} & 5000 & $0.791 \mathrm{E}-01$ \\
\hline & 10,000 & $0.559 \mathrm{E}-01$ \\
\hline \multirow{3}{*}{$3 \mathrm{kT} / 2 \Delta \mathrm{E}=0.234$} & 20,000 & $0.396 \mathrm{E}-01$ \\
\hline & 40,000 & $0.280 \mathrm{E}-01$ \\
\hline & 80,000 & $0.198 \mathrm{E}-01$ \\
\hline \multirow[t]{2}{*}{ CrIII $3 d^{3}\left({ }^{4} F\right) 4 s^{5} F-3 d^{3}\left({ }^{4} F\right) 4 p^{5} D^{o}$} & 5000 & $0.732 \mathrm{E}-01$ \\
\hline & 10,000 & $0.518 \mathrm{E}-01$ \\
\hline & 20,000 & $0.366 \mathrm{E}-01$ \\
\hline \multirow[t]{2}{*}{$3 \mathrm{kT} / 2 \Delta \mathrm{E}=0.234$} & 40,000 & $0.259 \mathrm{E}-01$ \\
\hline & 80,000 & $0.183 \mathrm{E}-01$ \\
\hline \multirow[t]{2}{*}{ CrIII $3 d^{3}\left({ }^{4} F\right) 4 s^{3} F-3 d^{3}\left({ }^{4} F\right) 4 p^{3} F^{o}$} & 5000 & $0.850 \mathrm{E}-01$ \\
\hline & 10,000 & $0.601 \mathrm{E}-01$ \\
\hline & 20,000 & $0.425 \mathrm{E}-01$ \\
\hline \multirow[t]{2}{*}{$3 \mathrm{kT} / 2 \Delta \mathrm{E}=0.258$} & 40,000 & $0.301 \mathrm{E}-01$ \\
\hline & 80,000 & $0.213 \mathrm{E}-01$ \\
\hline \multirow[t]{2}{*}{ CrIII $3 d^{3}\left({ }^{4} F\right) 4 s^{3} F-3 d^{3}\left({ }^{4} F\right) 4 p^{3} G^{o}$} & 5000 & $0.905 E-01$ \\
\hline & 10,000 & $0.640 \mathrm{E}-01$ \\
\hline & 20,000 & $0.453 \mathrm{E}-01$ \\
\hline \multirow{2}{*}{$3 \mathrm{kT} / 2 \Delta \mathrm{E}=0.258$} & 40,000 & $0.320 \mathrm{E}-01$ \\
\hline & 80,000 & $0.226 \mathrm{E}-01$ \\
\hline \multirow[t]{2}{*}{ CrIII $3 d^{3}\left({ }^{4} F\right) 4 s^{3} F-3 d^{3}\left({ }^{4} F\right) 4 p^{3} D^{o}$} & 5000 & 0.101 \\
\hline & 10,000 & $0.715 \mathrm{E}-01$ \\
\hline & 20,000 & $0.505 E-01$ \\
\hline \multirow{2}{*}{$3 \mathrm{kT} / 2 \Delta \mathrm{E}=0.258$} & 40,000 & $0.357 \mathrm{E}-01$ \\
\hline & 80,000 & $0.253 \mathrm{E}-01$ \\
\hline
\end{tabular}

The obtained Stark widths will be used for an example of the importance of the Stark broadening mechanism in stellar atmospheres. Hamdi et al. [48] and Dimitrijević et al. [26] demonstrated that the Stark broadening is usually larger than or comparable to Doppler broadening in atmospheres of DO white dwarfs, where effective temperatures are within the interval 45,000-120,000 K. Here we will extend this to the cool DO white dwarfs, marking the hot end of the so-called DB gap, corresponding to an interval in effective temperatures from 30,000-45,000 K [49].

In Figure 1 are shown logarithms of Stark (SW) and thermal Doppler (DW) widths for Cr III $3 \mathrm{~d}^{3}\left({ }^{4} \mathrm{~F}\right) 4 \mathrm{~s}^{5} \mathrm{~F}-3 \mathrm{~d}^{3}\left({ }^{4} \mathrm{~F}\right) 4 \mathrm{p}^{5} \mathrm{~F}^{o}(\lambda=2121.8 \AA)$ and $3 \mathrm{~d}^{3}\left({ }^{4} \mathrm{~F}\right) 4 \mathrm{~s}^{3} \mathrm{~F}-3 \mathrm{~d}^{3}\left({ }^{4} \mathrm{~F}\right) 4 \mathrm{p}^{3} \mathrm{D}^{o}(\lambda=2478.9 \AA)$ spectral lines as a function of Rosseland optical depth $\left(\tau_{\text {Ros }}\right)$, for an atmospheric model [50] with effective temperatures $\mathrm{T}_{\text {eff }}=40,000 \mathrm{~K}$, and $\log g=6$, where $g$ is surface gravity. These two lines were chosen since they have the smallest and largest Stark width, so for all other lines the corresponding curves will be between the curves presented in Figure 1. In Figure 2, Stark and thermal Doppler widths are presented for the same spectral lines, but for a model with $T_{\text {eff }}=40,000 \mathrm{~K}$, and $\log g=9$. We can see that there are large atmospheric layers where Stark broadening is dominant in comparison with thermal Doppler broadening. We can also see that the influence of Stark broadening increases with the increase of the 
value of Rosseland opacity, and that gravity becomes more important for atmospheric models with larger surface.

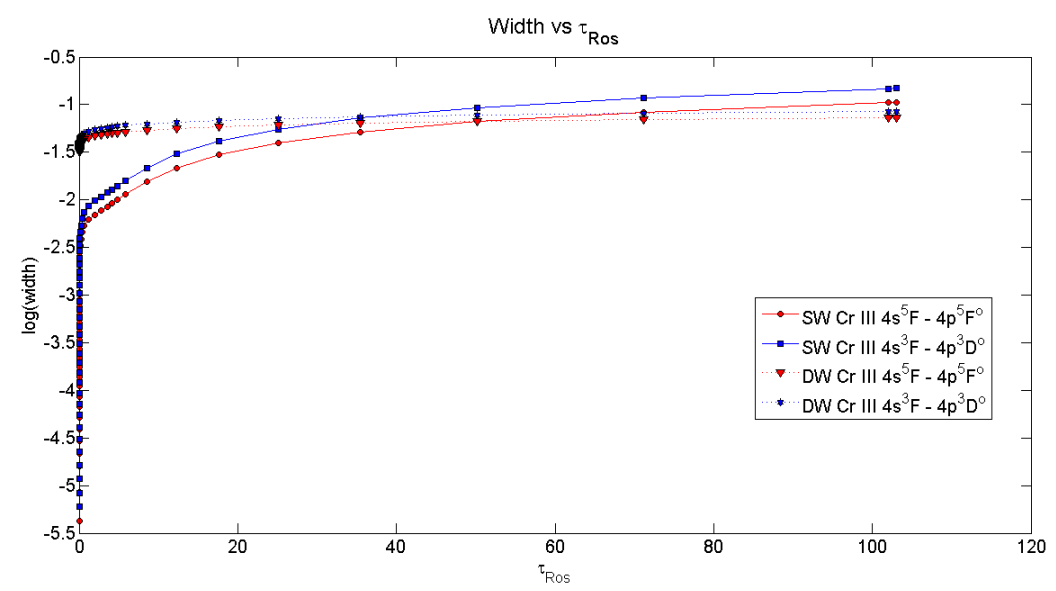

Figure 1. Stark (SW) and Doppler (DW) widths for Cr III $3 \mathrm{~d}^{3}\left({ }^{4} \mathrm{~F}\right) 4 \mathrm{~s}^{5} \mathrm{~F}-3 \mathrm{~d}^{3}\left({ }^{4} \mathrm{~F}\right) 4 \mathrm{p}^{5} \mathrm{~F}^{o}(\lambda=2121.8 \AA)$ and $3 \mathrm{~d}^{3}\left({ }^{4} \mathrm{~F}\right) 4 \mathrm{~s}^{3} \mathrm{~F}-3 \mathrm{~d}^{3}\left({ }^{4} \mathrm{~F}\right) 4 \mathrm{p}^{3} \mathrm{D}^{o}(\lambda=2478.9 \AA)$ spectral lines as a function of Rosseland optical depth $\left(\tau_{\text {Ros }}\right)$. Stark and Doppler widths are shown for an atmospheric model [50] with effective temperatures $\mathrm{T}_{\text {eff }}=40,000 \mathrm{~K}$, and $\log g=6$.

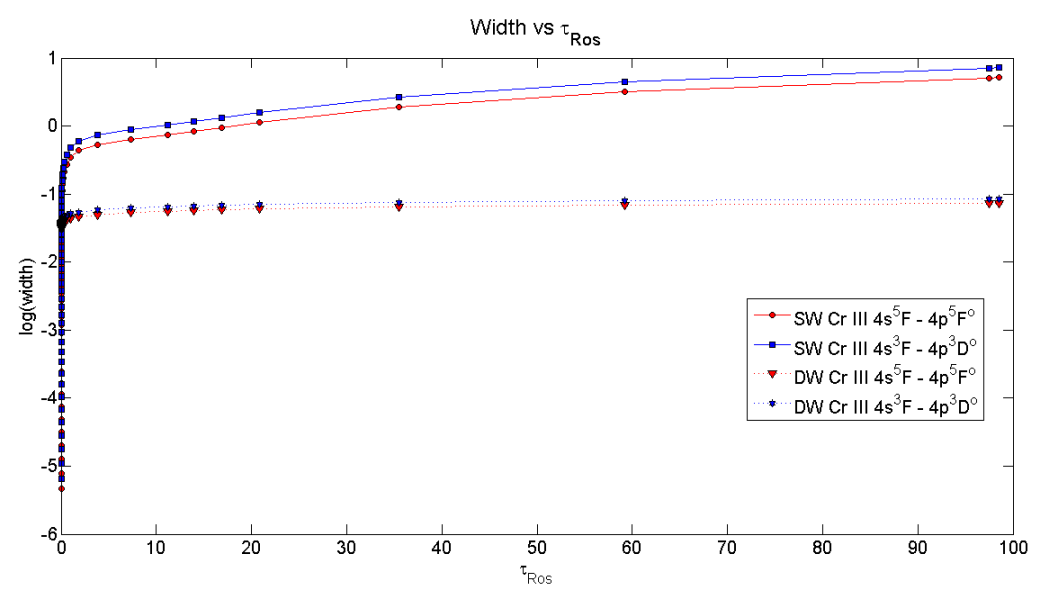

Figure 2. Stark (SW) and Doppler (DW) widths for Cr III $3 \mathrm{~d}^{3}\left({ }^{4} \mathrm{~F}\right) 4 \mathrm{~s}^{5} \mathrm{~F}-3 \mathrm{~d}^{3}\left({ }^{4} \mathrm{~F}\right) 4 \mathrm{p}^{5} \mathrm{~F}^{o}(\lambda=2121.8 \AA)$ and $3 \mathrm{~d}^{3}\left({ }^{4} \mathrm{~F}\right) 4 \mathrm{~s}^{3} \mathrm{~F}-3 \mathrm{~d}^{3}\left({ }^{4} \mathrm{~F}\right) 4 \mathrm{p}^{3} \mathrm{D}^{o}(\lambda=2478.9 \AA)$ spectral lines as a function of Rosseland optical depth $\left(\tau_{\text {Ros }}\right)$. Stark and Doppler widths are shown for an atmospheric model [50] with effective temperatures $\mathrm{T}_{\text {eff }}=40,000 \mathrm{~K}$, and $\log g=9$.

Seaton [51] underlines that Stark broadening is the dominant broadening mechanism in subphotospheric layers. If chromium exists in the stellar atmosphere, it also exists in various ionization stages in subphotospheric layers, since it is created in thermonuclear reactions in stellar interiors, and the corresponding Stark broadening parameters are useful for the theoretical consideration and modelling of such layers, as well as for the calculation of radiative transfer in such conditions even for cooler stars, where chromium is present but Stark broadening in the atmosphere is negligible.

The Stark widths of Cr III spectral lines, shown in Table 1, will be implemented in the STARK-B database [52,53], dedicated to the investigation, modelling, and diagnostics of the plasma of stellar atmospheres and for the diagnostics of laboratory plasmas, but also useful for investigation of laser-produced and inertial fusion plasma, as well as for plasma technologies. The STARK-B database is also included in the Virtual Atomic and Molecular Data Center (VAMDC) [54,55], created to enable an easier and more effective search of atomic and molecular data from different databases. 
Stark line widths calculated in this work also contribute to the creation of a set of such data for as large as possible a number of spectral lines, since this is important for a number of problems such as stellar spectra analysis and synthesis, opacity calculations, and the modelling of stellar atmospheres.

Acknowledgments: The support of the Ministry of Education, Science and Technological Development of the Republic of Serbia through project 176002 is gratefully acknowledged.

Author Contributions: The authors contributed equally to this work.

Conflicts of Interest: The authors declare no conflict of interest. The founding sponsors had no role in the design of the study; in the collection, analyses, or interpretation of data; in the writing of the manuscript, and in the decision to publish the results.

\section{References}

1. Underhill, A.B. The Ultraviolet Spectrum of Eta Canis Majoris, B5 Ia. Astrophys. J. Suppl. Ser. 1974, 27, 359-389.

2. Underhill, A.B. Line Identifications in the Ultraviolet Spectra of Tau Herculis (B5 IV) and Zeta Draconis (B6 III). Astrophys. J. Suppl. Ser. 1974, 34, 309-380.

3. Fahey, R.P.; Fischel, D.; Sparks, W. Intensity Changes in the UV Spectrum of Beta Cephei. NASCP 1982, 2238, 442-445.

4. Bauer, W.H.; Bennett, P.D. An Atlas of Far-ultraviolet Spectra of the Zeta Aurigae Binary 31 Cygni with Line Identifications. Astrophys. J. Suppl. Ser. 2014, 211, 27.

5. Johansson, S. On regularities in complex spectra of iron group elements and their dominance in stellar spectra. Phys. Scr. 1987, 36, 99-106.

6. Swings, J.P.; Klutz, M.; Peytremann, E.; Vreux, J.M. Fe III lines in the ultraviolet spectra of early B stars. Astron. Astrophys. Suppl. Ser. 1976, 25, 193-212.

7. Cowley, C.R.; Frey, M. On the completeness of the analysis of third and fourth spectra of the iron group, and the spectra of early B stars. Nucl. Instrum. Methods Phys. Res. B 1988, 31, 214-221.

8. Smillie, D.G.; Pickering, J.C.; Smith, P.L. Reference ultraviolet wavelengths of Cr III measured by Fourier transform spectrometry. Mon. Not. R. Astron. Soc. 2008, 390, 733-740.

9. Adelman, S.J.; Philip, A.G.D. Elemental abundances of field horizontal branch stars-IV. HD 74721, 86986 and 93329. Mon. Not. R. Astron. Soc. 1996, 280, 285-290.

10. Caliskan, H.; Adelman, S.J. Elemental abundance analyses with DAO spectrograms-XVII. The superficially normal early A stars 2 Lyncis, omicron Ursa Majoris and phi Aquilae. Mon. Not. R. Astron. Soc. 1997, 288, 501-511.

11. Adelman, S.J. Elemental abundance analyses with DAO spectrograms. VII-The late normal B stars Pi Ceti, 134 Tauri, 21 Aquilae, and Nu Capricorni and the use of RETICON spectra. Mon. Not. R. Astron. Soc. 1991, 252, 116-131.

12. Cowley, C.R.; Ryabchikova, T.; Kupka, F.; Bord, D.J.; Mathys, G.; Bidelman, W.P. Abundances in Przybylski's star. Mon. Not. R. Astron. Soc. 2000, 317, 299-309.

13. Schevchenko, V.S. V380 Ori: An Ae/Be Herbig star at an early stage of its envelope formation-emission lines of metals. Astron. Rep. 1994, 38, 505-519.

14. Andrievsky, S.M.; Kovtyukh, V.V.; Usenko, I.A. The chemical composition of the s-Cepheids. 1: Alpha Ursae Minoris (Polaris) and HR 7308 (V 473 Lyrae): Unique Cepheids of the Galaxy. Astron. Astrophys. 1994, 281, 465-470.

15. Goswami, A.; Rao, N.K.; Lambert, D.L. Emission-line spectra of XX Ophiuchi in 1996 and 1997. Observatory 2001, 121, 97-107.

16. Merrill, P.W. The Spectrum of XX Ophiuchi in 1949 and 1950. Astrophys. J. 1951, 114, 37-46.

17. Babel, J.; Lanz, T. Stratification of chemical elements in the atmosphere of the AP star 53 Camelopardalis. Astron. Astrophys. 1994, 281, 465-470. 1992, 263, 232-240.

18. Dimitrijević, M.S.; Ryabchikova, T.; Simić, Z; Popović, L.Č.; Dačić, M. The Influence of Stark broadening on Cr II spectral line shapes in stellar atmospheres. Astron. Astrophys. 2007, 469, 681-686.

19. Popović, L.Č.; Dimitrijević, M.S.; Tankosić, D. The stark broadening effect in hot star atmospheres: Au I and Au II lines. Astron. Astrophys. Suppl. Ser. 1999, 139, 617-623. 
20. Tankosić, D.; Popović, L.Č.; Dimitrijević, M.S. The electron-impact broadening parameters for Co III spectral lines. Astron. Astrophys. 2003, 399, 795-797.

21. Simić, Z.; Dimitrijević, M.S.; Popović, L.Č.; Dačić, M. Importance of collisions with charged particles for stellar UV line shapes: Cd III. Astron. Astrophys. 2004, 417, 375-380.

22. Simić, Z.; Dimitrijević, M.S.; Popović, L.Č.; Dačić, M. Stark broadening parameters for Cu III, Zn III and Se III lines in laboratory and stellar plasma. MNRAS 2006, 423, 766-773.

23. Dufour, P.; Ben Nessib, N.; Sahal-Bréchot, S.; Dimitrijević, M.S. Stark Broadening of Carbon and Oxygen Lines in Hot DQ White Dwarf Stars: Recent Results and Applications. Baltic Astron. 2011, 20, 511-515.

24. Larbi-Terzi, N.; Sahal-Bréchot, S.; Ben Nessib, N.; Dimitrijević, M.S. Stark-broadening calculations of singly ionized carbon spectral lines. New Astron. 2012, 12, 187-191.

25. Simić, Z; Dimitrijević, M.S.; Sahal-Bréchot, S. Stark broadening of resonant Cr II $3 d^{5}-3 d^{4} 4 p$ spectral lines. Mon. Not. R. Astron. Soc. 2013, 432, 2247-2251.

26. Dimitrijević, M.S.; Simić, Z.; Kovačević, A.; Valjarević, A.; Sahal-Bréchot, S. Stark broadening of Xe VIII spectral lines. Mon. Not. R. Astron. Soc. 2015, 454, 1736-1741.

27. Majlinger, Z.; Simić, Z.; Dimitrijević, M.S. Stark broadening of Zr IV spectral lines in the atmospheres of chemically peculiar stars. Mon. Not. R. Astron. Soc. 2017, 470, 1911-1918.

28. Gianninas, A.; Hermes, J.J.; Brown, W.R.; Dufour, P.; Barber, S.D.; Kilic Mukremin, B.; Kenyon, S.J.; Harrold, S.T. SDSS J074511.56+194926.5: Discovery of a Metal-rich and Tidally Distorted Extremely Low Mass White Dwarf. Astrophys. J. 2014, 781, 104.

29. Dufour, P.; Bergeron, P.; Schmidt, G.D.; Liebert, J.; Harris, H.C.; Knapp, G.R.; Anderson, S.F.; Schneider, D.P. Model Atmosphere Analysis of the Weakly Magnetic DZ White Dwarf G165-7. Astrophys. J. 2006, 651, 1112-1119.

30. Klein, B.; Jura, M.; Koester, D.; Zuckerman, B.; Melis, C. Chemical Abundances in the Externally Polluted White Dwarf GD 40: Evidence of a Rocky Extrasolar Minor Planet. Astrophys. J. 2010, 709, 950-962.

31. Rauch, T.; Ziegler, M.; Werner, K.; Kruk, J.W.; Oliveira, C.M.; Vande Putte, D.; Mignani, R.P.; Kerber, F. High-resolution FUSE and HST ultraviolet spectroscopy of the white dwarf central star of Sh 2-216. Astron. Astrophys. 2007, 470, 317-329.

32. Dimitrijević, M.S.; Ryabchikova, T.; Popović, L.Č.; Shulyak, D.; Khan, S. On the Influence of Stark broadening on Cr I lines in stellar atmospheres. Astron. Astrophys. 2005, 435, 1191-1198.

33. Sahal-Bréchot, S. Impact theory of the broadening and shift of spectral lines due to electrons and ions in a plasma. Astron. Astrophys. 1969, 1, 91-123.

34. Sahal-Bréchot, S. Impact theory of the broadening and shift of spectral lines due to electrons and ions in a plasma (continued). Astron. Astrophys. 1969, 2, 322-354.

35. Sahal-Bréchot, S.; Dimitrijević, M.S.; Ben Nessib, N. Widths and Shifts of Isolated Lines of Neutral and Ionized Atoms Perturbed by Collisions With Electrons and Ions: An Outline of the Semiclassical Perturbation (SCP) Method and of the Approximations Used for the Calculations. Atoms 2014, 2, 225-252.

36. Lakićević, I.S. Estimated Stark widths and shifts of neutral atom and singly charged ion resonance lines. Astrophys. J. 1951, 121, 37-41.

37. Kurucz, R. L. ATLAS9 Stellar Atmosphere Programs and $2 \mathrm{~km} \mathrm{~s}^{-1}$ grid, Kurucz CD-ROM No. 13; Smithsonian Astrophysical Observatory: Cambridge, MA, USA, 1993; pp. 13, 22-23.

38. Dimitrijević, M.S.; Simić, Z.; Sahal-Bréchot, S. On the Stark broadening of Cr VI spectral lines in astrophysical plasmas. J. Phys. Conf. Ser. 2017, 810, 012021.

39. Rathore, B.A.; Lakićević, I.S.; Ćuk, M.; Purić, J. Measurement of the Stark widths and shifts of Cr II $4 s^{4} \mathrm{D}-4 \mathrm{p}^{4} \mathrm{~F}^{o}$ spectral lines. Phys. Rev. A 1984, 28, 2264-2268.

40. Aguilera, J.A.; Aragón, C.; Manrique, J. Experimental Stark widths and shifts of Cr II spectral lines. Mon. Not. R. Astron. Soc. 2014, 438, 841-845.

41. Dimitrijević, M.S.; Konjević, N. Stark widths of doubly- and triply-ionized atom lines. J. Quant. Spectrosc. Radiat. Transf. 1980, 24, 451-459.

42. Dimitrijević, M.S.; Kršljanin, V. Electron-impact shifts of ion lines-Modified semiempirical approach. Astron. Astrophys. 1986, 165, 269-274.

43. Dimitrijević, M.S.; Popović, L.Č. Modified Semiempirical Method. J. Appl. Spectrosc. 2001, 68, 893-901.

44. Griem, H.R. Semiempirical Formulas for the Electron-Impact Widths and Shifts of Isolated Ion Lines in Plasmas. Phys. Rev. 1968, 165, 258-266. 
45. Sugar, J.; Corliss, C. Atomic Energy Levels of the Iron-Period Elements: Potassium through Nickel. J. Phys. Chem. Ref. Data 1985, 14 (Suppl. 2), 1-664.

46. Kramida, A.; Ralchenko, Y.; Reader, J.; NIST ASD Team. NIST Atomic Spectra Database; Version 5.3; National Institute of Standards and Technology: Gaithersburg, MD, USA, 2015.

47. Bates, D.R.; Damgaard, A. The Calculation of the Absolute Strengths of Spectral Lines. Phil. Trans. R. Soc. Lond. A 1949, 242, 101-122.

48. Hamdi, R.; Ben Nessib, N.; Milovanović, N.; Popović, L.Č.; Dimitrijević, M.S.; Sahal-Bréchot, S. Atomic data and electron-impact broadening effect in DO white dwarf atmospheres: SiVI. Mon. Not. R. Astron. Soc. 2008, $387,871-882$.

49. Chayer, P.; Vennes, S.; Dupuis, J.; Kruk, J.W. Abundance of Elements beyond the Iron Group in Cool DO White Dwarfs. Astrophys. J. Lett. 2005, 630, 169-172.

50. Wesemael, F. Atmospheres for hot, high-gravity stars. II. Pure helium models. Astrophys. J. Suppl. Ser. 1981, 45, 177-257.

51. Seaton, M.J. Atomic data for opacity calculations. I-General description. J. Phys. B 1987, 20, 6363-6378.

52. Sahal-Bréchot, S.; Dimitrijević, M.S.; Moreau, N. STARK-B Database, Observatory of Paris, LERMA and Astronomical Observatory of Belgrade. Available Online: http:/ / stark-b.obspm.fr (accesed on 1 February 2018).

53. Sahal-Bréchot, S.; Dimitrijević, M.S.; Moreau, N.; Ben Nessib, N. The STARK-B database VAMDC node: A repository for spectral line broadening and shifts due to collisions with charged particles. Phys. Scr. 2015, 50, 054008 .

54. Dubernet, M.L.; Boudon, V.; Culhane, J.L.; Dimitrijevic, M.S.; Fazliev, A.Z.; Joblin, C.; Kupka, F.; Leto, G.; Le Sidaner, P.; Loboda, P.A.; et al. Virtual atomic and molecular data centre. J. Quant. Spectrosc. Radiat. Transf. 2010, 111, 2151-2159.

55. Dubernet, M.L.; Antony, B.K.; Ba, Y.A.; Babikov, Y.L.; Bartschat, K.; Boudon, V.; Braams, B.J.; Chung, H.-K.; Daniel, F.; Delahaye, F.; et al. The virtual atomic and molecular data centre (VAMDC) consortium. J. Phys. B 2016, 49, 074003.

(C) 2018 by the authors. Licensee MDPI, Basel, Switzerland. This article is an open access article distributed under the terms and conditions of the Creative Commons Attribution (CC BY) license (http:/ / creativecommons.org/licenses/by/4.0/). 